\title{
The effect of near-earth thunderstorm electric field on the rate of the shower events detected by ARGO-YBJ
}

\author{
Kegu Axi, Xunxiu Zhou*, Bing Zhao, Daihui Huang, Huanyu Jia \\ On behalf of the ARGO-YBJ Collaboration ${ }^{\dagger}$ \\ School of Physical Science and Technology, Southwest Jiaotong University, Chengdu 610031, China \\ *E-mail: zhouxxeswitu.edu.cn
}

\begin{abstract}
The ARGO-YBJ detector was operated for 5 years at the YangBaJing Cosmic Ray Observatory (4300 $\mathrm{m}$ a. s. 1., Tibet, China). The high altitude location and the high granularity of the "full coverage" detector (central carpet with an active area of $\sim 93 \%$ ), allow a detailed space-time reconstruction of cosmic ray showers, making ARGO-YBJ well suitable to study the effects of thunderstorm electric field on the shower development in atmosphere. An accurate Monte Carlo simulation has been implemented to interpret the ARGO-YBJ data. CORSIKA is used to simulate the evolution and properties of extensive air showers in atmosphere. The G4argo code takes into account the response of the ARGO-YBJ detector. The correlation between the reconstructed shower event rate and the near-earth thunderstorm electric field has been studied in detail. We found that the variation of the shower rate depends on the strength and polarity of the electric field, and is also highly dependent on the zenith angle of the primary cosmic ray. These results could be helpful in understanding the effects of the electric fields on cosmic rays, and provide important information for other cosmic ray studies with ground-based detectors (such as LHAASO).
\end{abstract}

Keywords: Thunderstorm eletric field, Shower events, Monte Carlo simulations, Cosmic rays, ARGO-YBJ

36th International Cosmic Ray Conference -ICRC2019-

July 24th - August 1st, 2019

Madison, WI, U.S.A.

\footnotetext{
${ }^{*}$ Speaker.

${ }^{\dagger}$ for collaboration list see PoS(ICRC2019)1177.
} 


\section{Introduction}

Thunderclouds are distributed in the altitude range of $4-12 \mathrm{~km}$, and the electric field strength can be up to $2000 \mathrm{~V} / \mathrm{cm}[1,2]$. The influence of such strong field on charged particles cannot be ignored, so the effect of thunderstorm electric fields on cosmic ray secondary particles has always been a hot topic in atmospheric physics. In 1924, Wilson et al. [3] first suggested that strong electric fields could accelerate electrons to high energy. In 1992, Gurevich et al. [4] proposed an initial theory in which a "runaway" electron avalanche could cause dielectric breakdowns. In this mechanism, the electric field could accelerate electrons and if the energy is high enough, the electrons may become runaway and ionize the atmosphere molecules, giving rise to an avalanche process. Subsequently Babich et al. [5] and Marshall et al. [6] developed the theory now commonly referred to as "relativistic runaway electron avalanche" (RREA).

For decades, scientists have carried out many high altitude cosmic rays observations to detect the thunderstorm ground enhancement (TGE). In 1985, Alexeenko et al. [7] first found that the variation of the secondary cosmic ray flux was associated to the atmospheric electric field. Subsequently many TGEs were detected by ground-based experiments, such as EAS-TOP [8], ASEC [9], ARGO-YBJ [10], AS $\gamma$ [11], Mount Norikura [12]. These results showed that TGEs were correlated to thunderstorm electric fields, even if the possibility of other additional factors could not be completely excluded. At the same time, Monte Carlo simulations were performed to study the field effects on shower electrons. For example, Dwyer [13] and Symbalisty et al. [14] studied the threshold of the electric field strength $\left(E_{\mathrm{th}}\right)$ for RREA process and found that the value of $E_{\mathrm{th}}$ was dependent on the altitude.

The effect of atmospheric electric fields on secondary muons was also investigated. By using the Neutron Detector at Yangbajing, Wang et al. [15] found that the muons counting rate had no obvious correlations with the field, while Alexeenko et al. [16] observed a decrease of muons intensity during thunderstorms.

Interesting data were recently presented by Bartoli et al. [17], who observed a decrease in the single particle counting rate of the ARGO-YBJ detector in positive electric fields and small-scale enhancements in negative fields. Here, we define the positive electric field as one that accelerates positrons downward in the direction of the earth. By using simulations, a reasonable explanation of the phenomenon was given in their work.

During thunderstorms, the secondary charged particles in extensive air shower (EAS) are accelerated or decelerated according to their charge. The arrival time of shower particles in the detector, their position, their energy and flux will be modified by the field, producing variations in the detection rate. In this work, we performed Monte Carlo simulations by using the CORSIKA code to study the effect of near-earth thunderstorm electric fields on the intensity of the shower events detected by ARGO-YBJ. Our results may provide important information for physical analysis of the Large High Altitude Air Shower Observatory (LHAASO) [18] or other ground-based cosmic ray observatories.

\section{The ARGO-YBJ experiment}

The ARGO-YBJ experiment [19] was a fully covered ground EAS array located at the Yangbajing (YBJ) Cosmic Ray Observatory in Tibet, China, at an altitude of $4300 \mathrm{~m}$ above the sea level, corresponding to a vertical atmospheric depth of $606 \mathrm{~g} / \mathrm{cm}^{2}$. The detector is composed of a single layer of Resistive Plate Chambers (RPCs). The whole array consists of 154 unit named "clusters" of size $5.7 \times 7.6 \mathrm{~m}^{2}$ as shown in Fig. 1. Each cluster is made of 12 RPCs and each RPC is read out using 10 Pads (56 $\times 62 \mathrm{~cm}^{2}$ each). The array is composed of a central full-coverage carpet (130 clusters covering an area of $\left.77.85 \times 74.49 \mathrm{~m}^{2}\right)$ and an outer guard-ring (24 clusters reaching the area $111.26 \times 99.04 \mathrm{~m}^{2}$ ). The ARGO-YBJ detector is connected to two data acquisition 
systems, corresponding to the scaler and shower operation modes, which work independently. In scaler mode, the counting rate of each cluster is measured every $0.5 \mathrm{~s}[20,21]$. In shower mode, the detector is triggered when at least 20 pads in the central carpet are fired in a time window of 420 ns. The arrival time and location of each hit are recorded to reconstruct the shower front shape, the primary energy and the arrival direction [22].

During several thunderstorms, the rate of reconstructed shower has shown significant variations. In this work, Monte Carlo simulation has been performed to study the correlation between the flux variation of reconstructed showers and the near-earth thunderstorm electric field.

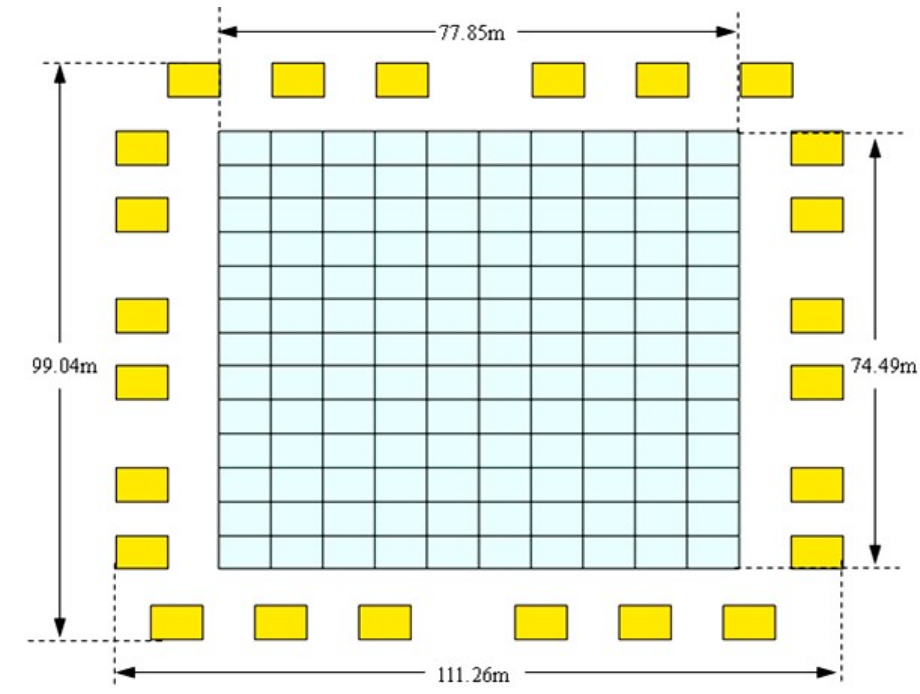

Fig. 1: ARGO-YBJ detector layout.

\section{Simulation paratmeters}

CORSIKA (COsmic Ray SImulations for KAscade) is a detailed Monte Carlo program to study the evolution and properties of extensive air showers in the atmosphere initiated by photons, protons, or nuclei of energies up to $10^{17} \mathrm{eV}$ [23]. The code of CORSIKA 7.5700, which takes into account the effect of the electric field on the transport of electrons and positrons, was used in this simulation work. The selected hadronic interaction models are QGSJETII-04 for high energy and GHEISHA for low energy events. We assume an electric field length of $1000 \mathrm{~m}$, extending from the detector level, $4300 \mathrm{~m}$, up to $5300 \mathrm{~m}$ (i.e. from atmospheric depth $606 \mathrm{~g} / \mathrm{cm}^{2}$ to $531 \mathrm{~g} / \mathrm{cm}^{2}$ ). The electric field intensity is chosen as a series of values in the range from -1000 to $1000 \mathrm{~V} / \mathrm{cm}$. Taking into account the energy threshold of the ARGO-YBJ detector [22], protons showers with energy range from $100 \mathrm{GeV}$ to $1 \mathrm{PeV}$ are selected as primary particles in the simulation, with a power law spectrum with index $\alpha=-2.7$. We consider protons with zenith angle in the interval from $0^{\circ}$ to $60^{\circ}$. The energy cutoff for secondary particles is set to $0.1 \mathrm{MeV}$, i.e. positrons and electrons with energy below this value are discarded from the simulation. The number of simulated showers is $2 \times 10^{4}$.

To simulate the transport of particles in the detector we used the G4argo code, a GEANT4based simulation package developed for the ARGO-YBJ detector [24]. In this simulation, the sampling area is set to $400 \times 400 \mathrm{~m}^{2}$. To increase the statistics of events, each shower arriving at the detector is sampled 100 times. 


\section{Simulation results}

\subsection{The electric field effects on the rate of reconstructed shower events}

The correlation between the electric field strength and the rate of reconstructed shower events detected by ARGO-YBJ was simulated. Fig. 2 shows the percent variation of shower events for different electric field values. In negative fields (accelerating electrons), the event rate increases as the field strength increases, with an enhancement up to $26.3 \%$ for fields of $-1000 \mathrm{~V} / \mathrm{cm}$. In positive fields (accelerating positrons), if the electric field strength is greater than $700 \mathrm{~V} / \mathrm{cm}$, the event number increases with increasing field with an enhancement up to $4.2 \%$ in field of $1000 \mathrm{~V} / \mathrm{cm}$. On the contrary, the number of events is reduced in the field range $0-700 \mathrm{~V} / \mathrm{cm}$ with a maximum variation of $-1.3 \%$.

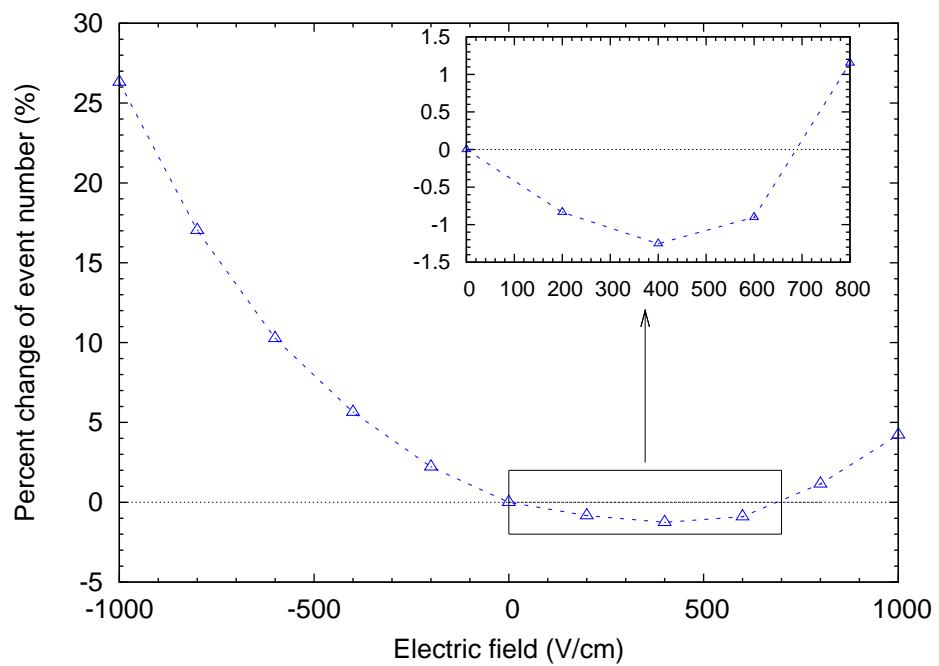

Fig. 2: Percent variation of the number of reconstructed showers as a function of the electric field in ARGOYBJ. The inset shows an enlarged view of the field range where the shower number is reduced.

To understand the rate variation shown in Fig. 2, it is useful to study the energy distributions of electrons and positrons, since the effect is due to the difference in the number and energy of electrons and positrons in the shower. This mechanism has been fully described in our previous works, where the variations of single particle rates in ARGO-YBJ were analyzed and successfully explained [17, 21]. According to the Bethe theory [25], the drag force increases with increasing energy when the positron/electron energy is larger than $\sim 1 \mathrm{MeV}$. This means that the electric field has a larger effect on charged particles with smaller energy. The number of electrons at YBJ is in average 1.7 times larger than that of positrons. Fig. 3 shows the ratio of electrons to positrons $\left(N_{e^{-}} / N_{e^{+}}\right)$with different energy. The ratio becomes larger with decreasing energy. The energy distribution of positrons and electrons in absence of electric field is shown in Fig. 4. In the energy region 1-18 MeV, the proportion of electrons (i.e. the ratio of electrons in a certain energy region to the total number of electrons) is larger than that of positrons. The phenomenon is reversed in higher energy regions. Since positrons have a larger energy than electrons and are in a smaller number, the increase of positrons cannot compensate for the decrease of electrons in positive fields when the field strength is smaller than $700 \mathrm{~V} / \mathrm{cm}$, and so a reduction of the total number of electrons and positrons occurs. ARGO-YBJ detects electrons and positrons. So it is easy to understand the rate variations shown in Fig. 2, and in particular the decrease phenomenon in the field range $0-700$ $\mathrm{V} / \mathrm{cm}$. 


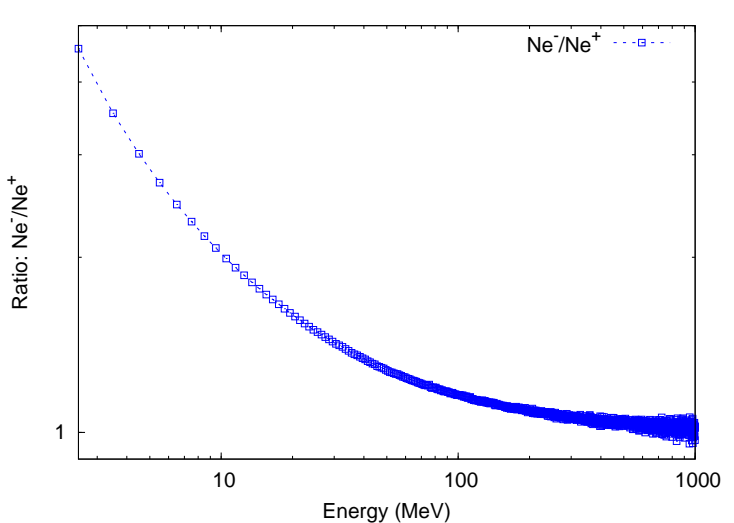

Fig. 3: Ratios of electrons to positrons as a function of energy in absence of electric field.

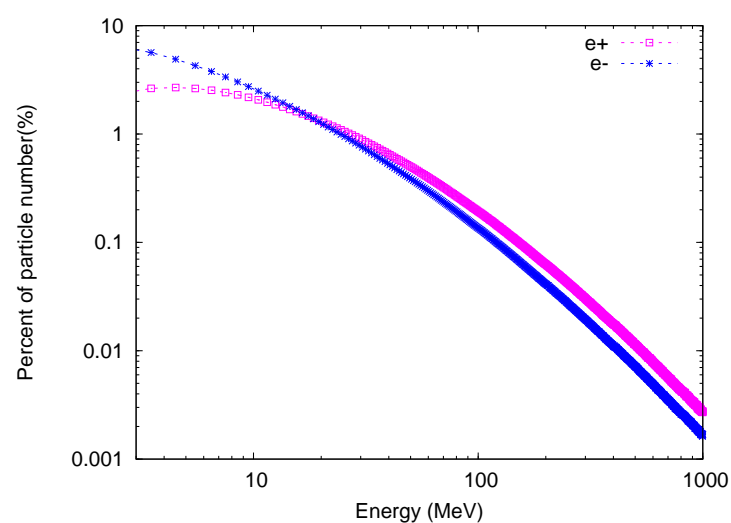

Fig. 4: Percent distributions of electrons and positrons as a function of energy in absence of electric field.

\subsection{The field effects on the reconstructed events with different primary zenith angles}

The thickness of the atmospheric layer varies with the zenith angles of the primary particle, so the field effects on the secondary charged particles is different. Fig. 5 describes the percentage variations of the reconstructed shower rate as a function of the primary zenith angle in a field strength of $-1000 \mathrm{~V} / \mathrm{cm}$ and $1000 \mathrm{~V} / \mathrm{cm}$. In a field of $-1000 \mathrm{~V} / \mathrm{cm}$, the number of events increases, and the enhancement amplitude decreases with increasing zenith angle. For zenith angle of $5^{\circ}$ and $55^{\circ}$, the increase is $30.6 \%$ and $8.7 \%$, respectively. In a field of $1000 \mathrm{~V} / \mathrm{cm}$, the number of showers increases for $\theta<40^{\circ}$, but decreases for $\theta>40^{\circ}$. The variation is $8.0 \%$ for $=5^{\circ}$ and $-8.6 \%$ for $\theta=55^{\circ}$. From Fig. 5, we can see that the percent variation of reconstructed showers is strongly dependent on the primary zenith angle and the polarity of the electric field.

Fig. 6 shows the variation of the number of reconstructed showers with different primary zenith angles in different fields. We can see that the variation is correlated with the polarity and strength of the electric field, and it is also highly dependent on the zenith angle of the primary particle. In negative field, the number increases as the field strength increases, and the enhancement becomes larger at smaller zenith angles. In positive fields, if $\theta>40^{\circ}$, the number of showers decreases for any field strength. If $\theta<40^{\circ}$, the number of showers decreases in field of strength 0 $-700 \mathrm{~V} / \mathrm{cm}$, but increases when the field is above $700 \mathrm{~V} / \mathrm{cm}$.

To understand this phenomenon, we simulate and study the field effect on the lateral distribution of secondary positrons and electrons at YBJ. We found that the lateral distribution widens during thunderstorms and this effect increases with zenith angle. The variation of the lateral distribution, combined with the finite size of the detector, produces the effects shown in Fig. 5 and 6 (see [26] for further details).

\subsection{The field effects on the reconstructed shower events with different $N_{\text {pad }}$}

$N_{\text {pad }}$ (pad-multiplicity) is the number of fired pads of the shower event recorded by the detector. Fig. 7 shows the variation of the number of reconstructed showers as a function of $N_{\text {pad }}$ in a field with strength $1000 \mathrm{~V} / \mathrm{cm}$. We can see that the effect is almost independent from $N_{\text {pad }}$, i.e. the rate of small showers and large showers vary in a similar way. The differences in variation are less than $1.8 \%$ for fields of $-1000 \mathrm{~V} / \mathrm{cm}$ and $0.4 \%$ for fields of $1000 \mathrm{~V} / \mathrm{cm}$.

The same result is shown in Fig. 8, where the variations of the number of showers with different $N_{\text {pad }}$ as a function of electric field are presented. For different $N_{\text {pad }}$, the variation amplitudes are almost the same. 


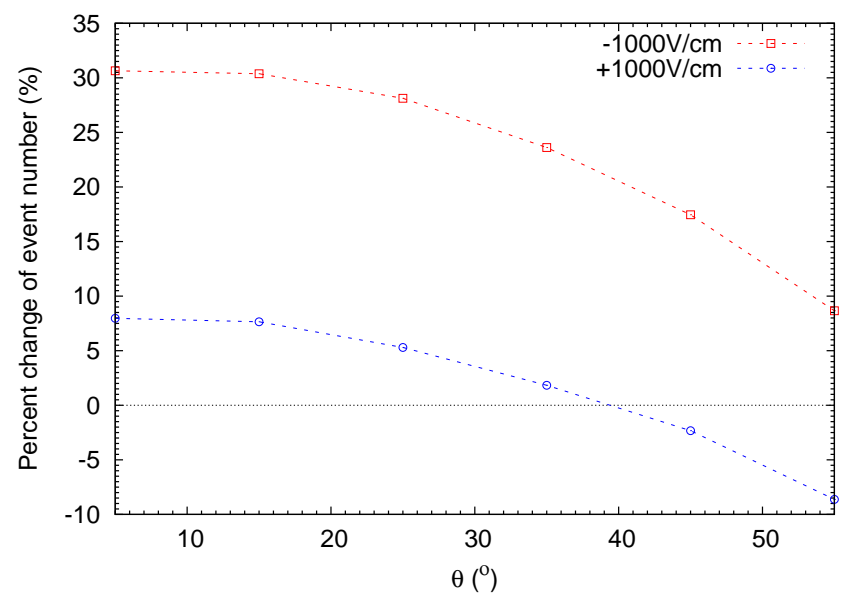

Fig. 5: Percent variation of reconstructed showers as a function of the primary zenith angle for electric field of $\pm 1000 \mathrm{~V} / \mathrm{cm}$.

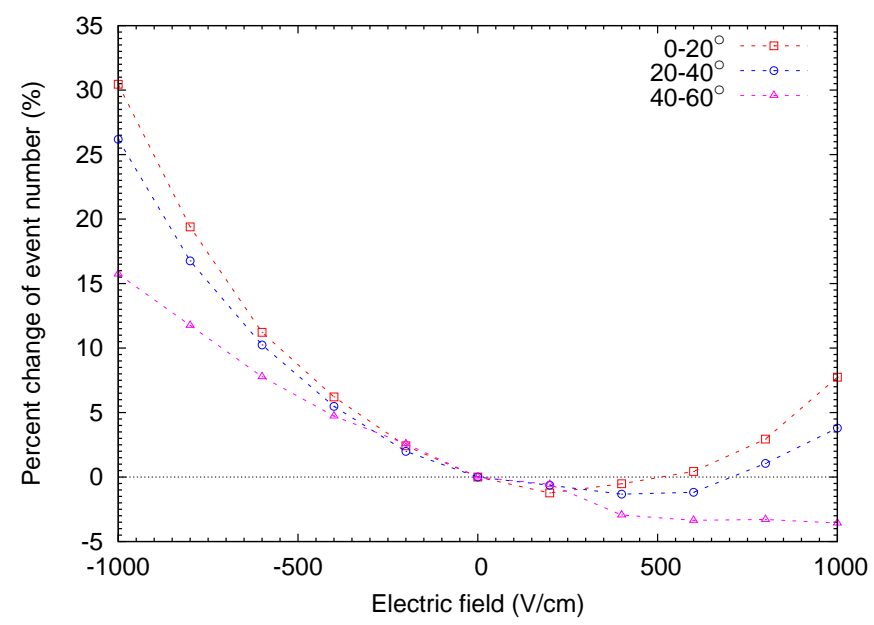

Fig. 6: Percent variations of reconstructed showers as a function of electric field with different primary zenith angles.

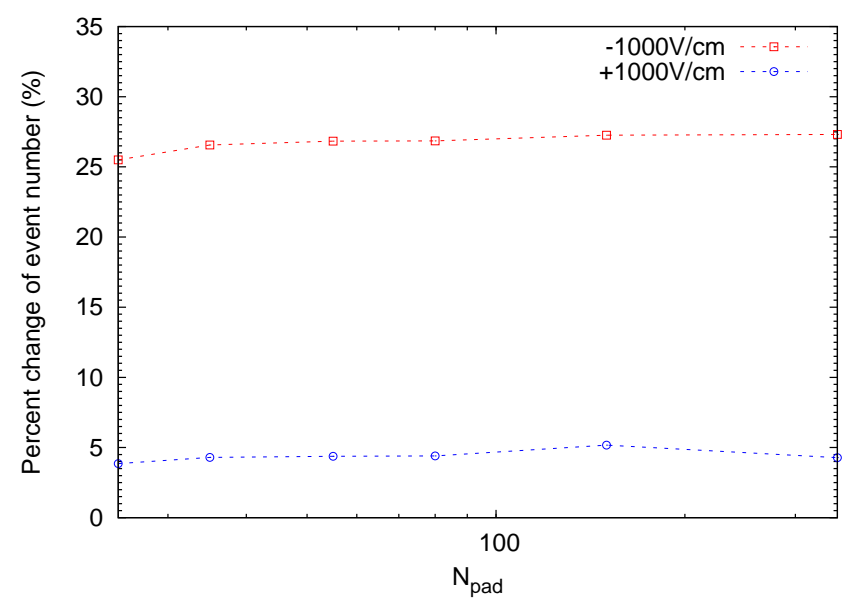

Fig. 7: Percent variation of reconstructed showers as a function of $N_{\text {pad }}$ for electric field of $\pm 1000 \mathrm{~V} / \mathrm{cm}$. 


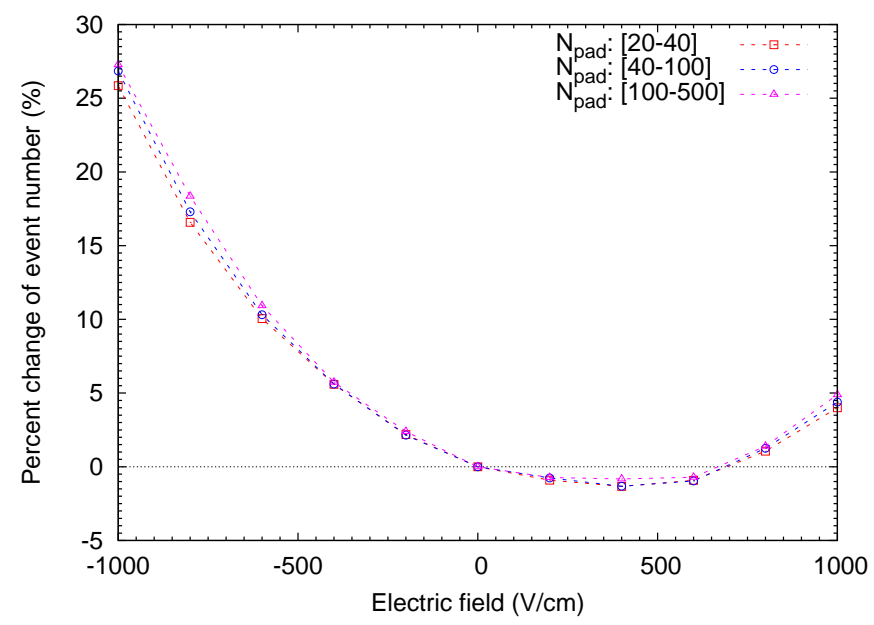

Fig. 8: Percent variation of reconstructed showers as a function of the field, for different values of $N_{\text {pad }}$.

\section{Conclusions}

Monte Carlo simulations have been performed to study the effect of thunderstorm electric fields on the rate of reconstructed showers detected by ARGO-YBJ. The number of events increases or decreases according to the field strength and polarity. In negative fields, the number of events increases with the electric field strength, with an amplitude up to $26.3 \%$ in field of $-1000 \mathrm{~V} / \mathrm{cm}$. In positive fields, if the field strength is greater than $700 \mathrm{~V} / \mathrm{cm}$, the number of showers increases with a variation reaching $4.2 \%$ for fields of $1000 \mathrm{~V} / \mathrm{cm}$, while the number of showers decreases in the field range $0-700 \mathrm{~V} / \mathrm{cm}$, with a maximum amplitude of $-1.3 \%$.

We also study the variations of the rate of reconstructed showers with different $N_{\text {pad }}$ and zenith angles. The results show that the correlation between the rate variation and $N_{\text {pad }}$ is not obvious. Instead, the rate variations are highly dependent on the primary zenith angle. With smaller zenith angles, the rate increase becomes larger in negative fields. In positive fields, the decrease becomes larger with higher zenith angles.

A similar rate increase/decrease, depending on the field strength and polarity, was indeed observed in the ARGO-YBJ single particle data and explained with the same mechanism [17]. The agreement between our previous analysis and simulations makes us confident that the EAS variations could also be due to the same effect. The analysis of EAS ARGO-YBJ data is currently in progress.

These results could be helpful to understand the phenomenon detected by ground-based experiments during thunderstorms, and useful to study the acceleration mechanism of secondary charged particles caused by atmospheric electric field.

\section{Acknowledgments}

This work is supported by the National Key R\&D Program of China under the grant No. 2018YFA0404201, the National Natural Science Foundation of China (NSFC) under Grants No. 11475141 and No. 11847307.

\section{References}

[1] D. X. Liu et al., Characteristics of lightning radiation source distribution and charge structure of squall line, Acta Physica Sinica, 62, 21 (2013). 
[2] M. Stolzenburg et al., Electric field values observed near lightning flash initiations, Geophysical Research Letters, 34, 4 (2007).

[3] R. C. T. Wilson, The electric field of a thundercloud and some of its effects, Proceedings of the Physical Society of London, 37, 32D (1924).

[4] A. V. Gurevich et al., Runaway electron mechanism of air breakdown and preconditioning during a thunderstorm, Physics Letters A, 165, 56 (1992).

[5] L. P. Babich et al., New data on space and time scales of relativistic runaway electron avalanche for thunderstorm environment: Monte Carlo calculations, Physics Letters A, 245, 5 (1998).

[6] T. C. Marshall et al., Electric field magnitudes and lightning initiation in thunderstorms, Journal of Geophysical Research Atmospheres, 100, D4 (1995).

[7] V. V. Alexeenko et al., Short perturbations of cosmic ray intensity and electric field in atmosphere, in: Proceedings $19^{\text {th }}$ ICRC, La Jolla, USA, Augest 11-23, 5 (1985).

[8] S. Vernetto for EAS-TOP Collaboration, The EAS counting rate during thunderstorms, in: Proceedings of $27^{\text {th }}$ ICRC, Copernicus Gesellschaft, Hambeg, Germany, Augest 7-15, 10 (2001).

[9] A. Chilingarian at el., Statistical analysis of the Thunderstorm Ground Enhancements (TGEs) detected on Mt. Aragats, Advances in Space Research,52, 6 (2013).

[10] Y. Zeng et al., Correlation between cosmic ray flux and electric atmospheric field variations with the ARGO-YBJ experiment, in: Proceedings of $33^{\text {rd }}$ ICRC, Rio de Janeiro, Brazil, July 2-9, 0757 (2013).

[11] H. Tsuchiya et al., Observation of thundercloud-related gamma rays and neutrons in Tibet, Physical Review D, 85, 9 (2012).

[12] H. Tsuchiya et al., Observation of an energetic radiation burst from mountain-top thunderclouds, Physical Review Letters, 102, 25 (2009).

[13] J. R. Dwyer et al., A fundamental limit on electric fields in air, Geophysical Research Letters, 30, 20 (2003).

[14] E. M. D. Symbalisty et al., Finite volume solution of the relativistic Boltzmann equation for electron avalanche studies, IEEE Transactions on Plasma Science, 26, 5 (1998).

[15] J. F. Wang et al., Effect of thunderstorm electric field on intensity of cosmic ray muons, Acta Physica Sinica, 61, 15 (2012).

[16] V. V. Alexeenko et al., Transient variations of secondary cosmic rays due to atmospheric electric field and evidence for pre-lightning particle acceleration, Physics Letters A, 301, 3-4 (2012).

[17] B. Bartoli et al., Observation of the thunderstorm-related ground cosmic ray flux variations by ARGO-YBJ, Physical Review D, 97, 4 (2018).

[18] Z. Cao, Status of LHAASO updates from ARGO-YBJ, Nuclear Instruments and Methods in Physics Research Section A: Accelerators, Spectrometers, Detectors and Associated Equipment, 742, $95-98$ (2014).

[19] G. K. Parks et al., Xray enhancements detected during thunderstorm and lightning activities, Geophysical Research Letters, 8, 11 (2013).

[20] T. D. Girolamo et al., Search for gamma ray bursts with the ARGO-YBJ detector in scaler mode, Nuclear Instruments and Methods in Physics Research Section A: Accelerators, Spectrometers, Detectors and Associated Equipment, 588, 1-2 (2008).

[21] X. X. Zhou et al., Effect of near-earth thunderstorms electric field on the intensity of ground cosmic ray positrons/electrons in Tibet, Astroparticle Physics, 84, 107 (2016).

[22] B. Bartoli et al. (ARGO-YBJ Collaboration), TeV gamma-ray survey of the northern sky using the ARGO-YBJ detector, Astrophys. J., 779, 27 (2013).

[23] J. N. Capdevielle et al., Extensive air shower simulations with the CORSIKA program, in: Aip Conference, American Institute of Physics, Jan 1, 276, 545 (1992).

[24] Y. Q. Guo et al., ARGO-YBJ detector simulation using GEANT4, Chinese Physics C, 34, 5 (2010).

[25] H. A. Bethe, Theory of passage of swift corpuscular rays through matter, Annals of Physics, 5, 325 (1930).

[26] K. G. Axi et al., The effect study of thunderstorm electric field on the lateral distribution of positrons and electrons at YBJ, in: Proceedings of $36^{\text {th }}$ ICRC, PoS(ICRC2019) 496. 